\title{
The Use of Benlate for Distinguishing Between Haploid and Diploid Strains of Aspergillus nidulans and Aspergillus terreus
}

\author{
By A. UPSHALL, B. GIDDINGS AND I. D. MORTIMORE \\ Department of Biological Sciences, University of Lancaster, Lancaster LAI ${ }_{4} Y Q$
}

(Received I December 1976)

\section{INTRODUCTION}

For accurate deductions to be drawn from the results of a parasexual analysis it is mportant to distinguish between the three classes of stable segregants, namely haploids, non-disjunctional diploids and cross-over diploids. With the well analysed Aspergillus nidulans this is not too difficult if one makes use of strains carrying diploid recognition systems (Käfer, Marshall \& Cohen, 1976), but where these systems are not available the major criterion for distinguishing ploidy is that of spore diameter, diploid strains having larger asexual spores because of the increase in nuclear volume. In the imperfect fungus Aspergillus terreus the haploid conidiospore diameter is between 1.8 and $2.4 \mu \mathrm{m}$ (Raper \& Fennell, 1965) and in our investigations with this fungus we have found it extremely difficult to classify unambiguously the ploidy of segregants by this technique.

Following the work of Hastie (1970), Kappas, Georgopoulos \& Hastie (1974) and Edwards, Normansell \& Holt (I975), the fungicide methyl-I-(butylcarbamoyl)-2-benzimidazole carbamate (benomyl or Benlate) is now the most widely used haploidizing agent for $A$. nidulans and we have found it to be efficient for $A$. terreus. For both species, the growth pattern of the colonies arising after mass conidial transfers of a diploid strain on to fungicide-containing medium is irregular, with initial retardation of growth linked to the production of slightly more vigorous, usually haploid, sectors. We felt that this growth pattern might prove a valuable method of distinguishing ploidy in $A$. terreus in which diploids would exhibit the marked instability whereas haploids would remain relatively stable. However, in a reconstruction experiment we found, surprisingly, that the ploidy difference could be distinguished after $36 \mathrm{~h}$ incubation on the fungicide medium with diploid colonies showing sensitivity whilst haploids were tolerant. This distinction could also be made between haploid and diploid strains of $A$. nidulans.

It was possible that the phenotypic difference was the result of recessive non-allelic mutations to Benlate tolerance within the haploid strains such that when combined in the diploid, the sensitive phenotype was expressed on fungicide-containing medium. This paper reports experiments on $A$. nidulans which reject this possibility and confirm that in $A$. nidulans, and by extrapolation in $A$. terreus, haploid strains are more tolerant to the fungicide than diploids. This difference provides an easy way of classifying the ploidy of segregants from both sexual and parasexual analyses. 
Table I. Genotypes of haploid A. nidulans strains used

Fungal Genetics Stock Centre codes are shown in parentheses. For gene designations, see Clutterbuck (1974), with the exception of acuF238 (Armitt, McCullough \& Roberts, 1976) and $h f a A I$ which defines high frequency of aneuploids (Mortimore \& Upshall, unpublished).

Lancaster code

$2(187)$
$9(375)$
86
90
$213 \quad(466)$
230
233
267

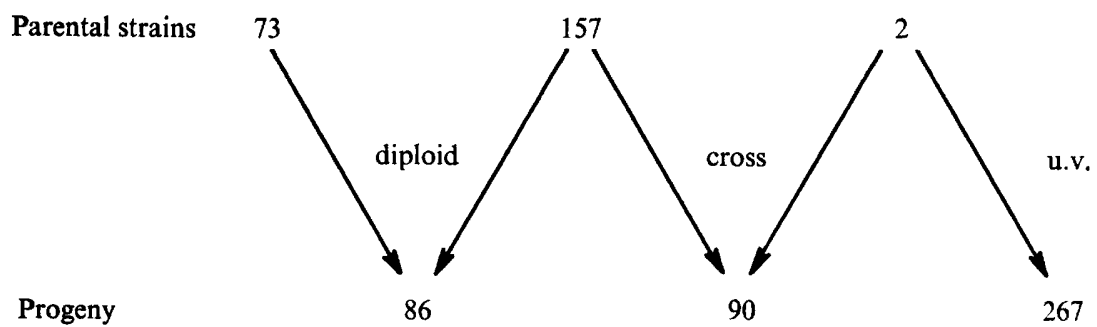

Fig. I. Pedigree of closely related $A$. nidulans strains (Lancaster codes) used in the analyses. Strain 73 has the genotype suAradE2o yA2 adE2o; wA3; galAr; pyroA4; facA303; sB3; nicB8; riboB2. Strain 157 has the genotype biAI $h f a A I$. For genotypes of other strains and abbreviations, see Table I.

\section{METHODS}

Strains. The A. nidulans strains directly involved in the analysis are listed in Table I: all are descendants of the original Glasgow strain but four have relatively close pedigree relationships (Fig. I). The $A$. terreus strains are listed in Table 2: all are derivatives of a wild-type isolate kindly provided by $\mathrm{Dr}$ C. E. Caten. No mutagenic treatments were involved in the recovery of the $A$. terreus mutant strains (Giddings \& Upshall, 1975) and hence the assumption can be made that, with the exception of the known mutations, the strains are isogenic.

Media and techniques. These were standard as for A. nidulans (Pontecorvo et al., 1953; Barratt, Johnson \& Ogata, r965). Benlate (Du Pont) was used as the commercial grade preparation and since we have found this an extremely efficient haploidizing agent we have not inquired as to its purity. In all experiments the Benlate powder was incorporated as a sterile aqueous suspension into molten complete medium to a final concentration of $\mathrm{I} .5$ p.p.m. The 'Benlate test' for distinguishing ploidy was carried out as follows:

After purification, master plates of the recombinant progeny were replicated on to a differential set of media to determine gene segregation patterns. Simultaneously these progeny were replicated on to complete medium incorporating Benlate and incubated at $37^{\circ} \mathrm{C}$. Provisional ploidy classification was made $36 \mathrm{~h}$ later. For the $A$. nidulans experiments this classification was confirmed by carrying out one or more alternative diploid identification procedures, namely (i) measurement of conidiospore diameter (Pontecorvo et al., 1953); (ii) examination of pigment and conidial head morphology differences (e.g. Croft 
Table 2. Genotypes of haploid A. terreus strains used

For identification of markers, see Giddings \& Upshall (1975).

Lancaster code

$\begin{array}{ll}\text { T6 } & \text { adeAI acrAI } \\ \text { T7 } & \text { whiAI; metBI } \\ \text { TI0* } & \text { bufAI } ; \text { BI } \\ \text { T12 } & \text { whiAI } ; \text { niaDI } \\ \text { T18 } & \text { sBI } \\ \text { T29 } & \text { adeAI acrAI; whiAI; metAI } \\ \text { T30* } & \text { sCI } ; \text { niaDI; bufAI }\end{array}$

* Previously designated creAI in Giddings \& Upshall (1975).

\& Jinks, I973); (iii) heterozygosity of the acrAI gene (Roper \& Käfer, I957); (iv) somatic instability and production of haploid sectors on prolonged incubation of the Benlate test plates.

For three $A$. nidulans combinations ( 2 and $230 ; 230$ and $267 ; 86$ and 90 ) both sexual and parasexual analyses were carried out; for two other combinations ( 9 and $90 ; 213$ and 233) only a parasexual analysis was undertaken. In the meiotic analyses at least I 25 randomly selected progeny were tested on the Benlate medium and in the parasexual analyses at least 25 segregants were tested. Within the parasexual analyses segregants were obtained from two sources: (i) spontaneously via the selection of conidial heads expressing recessive spore colour; and (ii) after growth of the diploid on complete medium plus Benlate. The somatic segregants for $A$. terreus were obtained in the same way but in this fungus the only unambiguous alternative test for ploidy was to allow prolonged incubation of the Benlate test plates.

\section{RESULTS AND DISCUSSION}

In the initial reconstruction experiments carried out with both fungi, a clear colonial difference was observed after $36 \mathrm{~h}$ (Fig. $2 a, c$ ), the haploid strain having grown appreciably more than the diploid. After prolonged incubation, the diploids became unstable and produced sectors exhibiting recessive spore colour whilst the haploids remained stable (Fig. $2 b, d$ ).

The applicability of this Benlate test depends on each haploid strain not carrying a gene mutation conferring tolerance to Benlate, such a mutation in one strain being non-allelic to a similar mutation in another. This was investigated using $A$. nidulans strains, sexual crosses of which can easily identify such a situation. If non-allelic tolerance mutations were present in each haploid strain a proportion of the haploid progeny should exhibit sensitivity to the fungicide, this proportion depending on the linkage relationships between any such genes. For the three crosses analysed, no Benlate-sensitive progeny were recovered. All progeny were Benlate tolerant to the same degree, and to the same degree as the parental strains. One can therefore deduce that these strains did not differ by non-allelic mutations to Benlate tolerance. The above result could have been obtained if the two strains carried allelic mutations to tolerance, but the pedigrees of our strains make this unlikely. Hence while two (strains 86 and 90) or three (strains 2, 90, 267) were closely related (Fig. I) and so might carry allelic mutations to tolerance, the remaining three (strains 2 I3, 230 and 233) have quite widely divergent pedigrees and are therefore unlikely to carry allelic mutations. Our conclusion is further supported by the observations that induced gene mutations to Benlate resistance are scattered throughout the genome (Clutterbuck, 1974). 

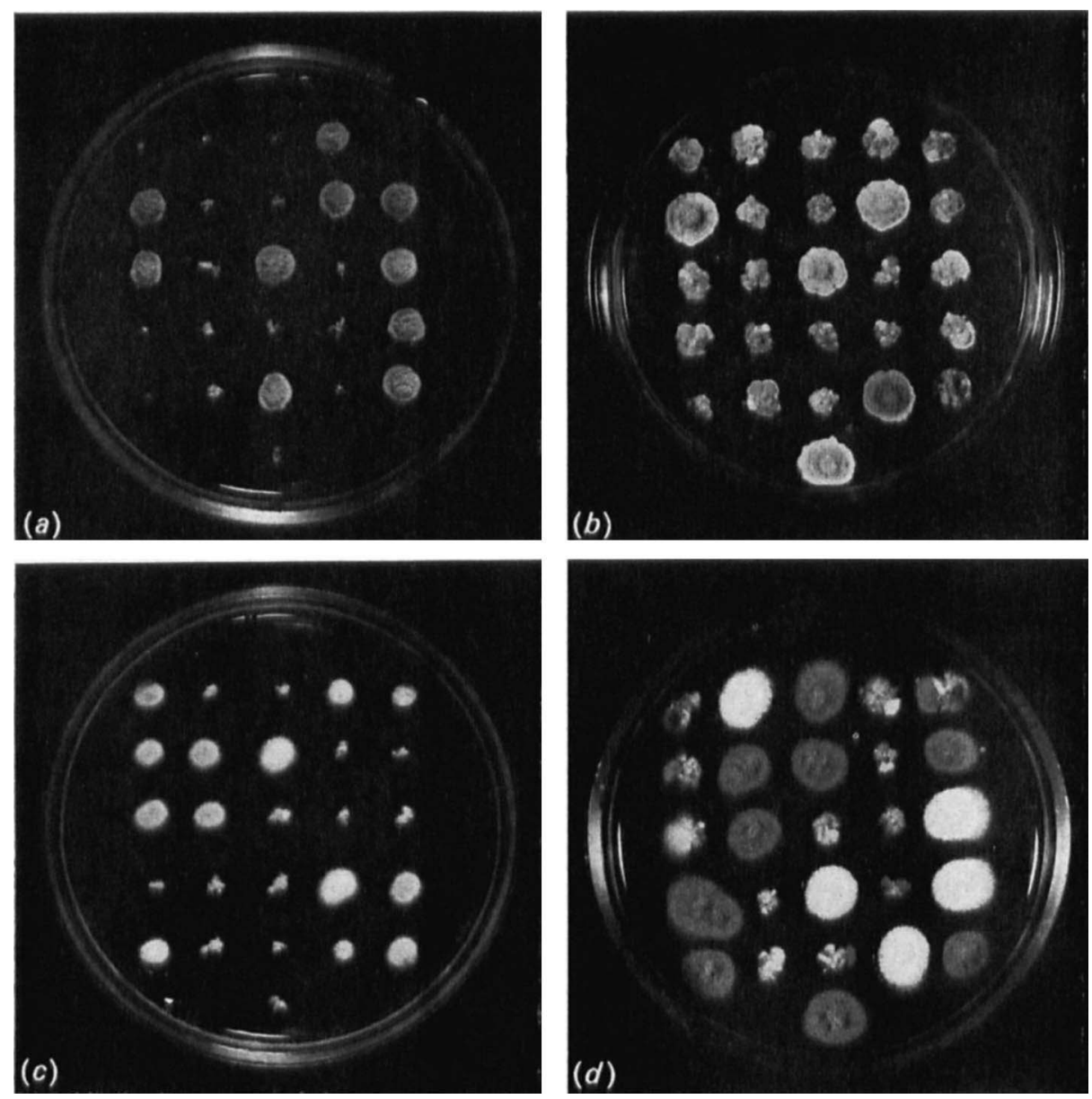

Fig. 2. Reconstruction experiment illustrating the growth responses of haploid and diploid strains of $A$. nidulans $(a, b)$ and $A$. terreus $(c, d)$ replicated on to complete medium plus Benlate (I.5 p.p.m.). $(a, c)$ After $36 \mathrm{~h}$ incubation at $37^{\circ} \mathrm{C}$ : diploids are small and haploids large. $(b, d)$ After 3 days incubation at $37^{\circ} \mathrm{C}$ : diploids are unstable and recessively coloured sectors appear while haploids remain stable. (The pattern of haploid and diploid colonies is not the same on all plates.)

Supporting evidence also comes from the parasexual analyses of the five diploid strains. These diploids were all more sensitive to Benlate than their parental haploid strains, and to the meiotic progeny from the three crosses. The parasexual segregants were provisionally classified as diploid or haploid on the basis of their growth pattern on the Benlate medium. In this situation sensitive haploid progeny could arise if the parents differed by recessive mutations to tolerance located on separate linkage groups. This possibility is an important consideration for the analysis of those two diploids where a sexual cross had not been carried out between the two haploid parents. Therefore, to check our provisional classification, the alternative diploid recognition tests were carried out as described in Methods. In all cases, these tests confirmed our provisional ploidy classification made on the Benlate test plates.

Clearly, tolerance of the fungicide is a function of the haploid nature of the genome, and this technique provides a convenient and rapid method for distinguishing ploidy. 
This is of importance since Benlate is known to yield a relatively high frequency of nondisjunctional diploid segregants (Kappas et al., 1974) and non-disjunctional diploids occur among spontaneous somatic recombinants (Pontecorvo \& Käfer, I958). These nondisjunctional diploid segregants may be phenotypically confused with the other two segregant classes since their conidiospore colour and growth responses on differential media can be the same. For example, spontaneous yellow recombinants were isolated from the diploid formed between strains $2 \mathrm{I} 3$ and 233, where all markers except pyroA4 and chaAI are located on mitotic linkage group I. Five (out of a total of 25) expressed the mutant genes gal ribo an pro lys and paba. By replicating on to the Benlate medium, we rapidly identified three of these as non-disjunctional diploid and two as haploid segregants.

These thorough experiments are not possible with the imperfect $A$. terreus. Nevertheless, the close similarity of the growth responses of the known haploid and diploid strains on the Benlate medium to $A$. nidulans leads us to conclude that the ploidy recognition test as described here is applicable and that, although closely related, the $A$. terreus strains do not differ by mutations to Benlate tolerance.

Because of the clarity of the haploid/diploid difference, it was considered that Benlate medium might enable a direct estimation of the frequency of spontaneous diploid progeny among ascospore samples of $A$. nidulans estimated to occur at a frequency of approximately $2 \%$ (Pritchard, 1954). To test this hypothesis, random ascospore samples of three different translocation-free crosses were spread over the surface of complete medium plus Benlate. In no case was a clear classification into two phenotypes possible. Instead, a spectrum of colony morphologies was observed ranging from extremely small unstable types to large normal phenotypes. Control platings on complete medium plus sodium deoxycholate (Mackintosh \& Pritchard, 1963) yielded no abnormal morphologies and all of a sample of 250 tested on Benlate medium were haploid. We believe this variable expression on the spread Benlate plates to be a reflexion of the disruption of nuclear division at different stages in development. Hence interference with the first nuclear division at germination will yield a thallus containing only aneuploid nuclei so generating the minute unstable colonies, whilst interference with nuclear division at successively later stages increases the proportion of haploid nuclei in the thallus giving the more normal phenotypes. Therefore, the value of the Benlate test for differentiating ploidy most likely relies on the point transfer of large numbers of conidia via the needle replicator, and it is unlikely to be of use in single spore analysis.

We wish to thank Drs C. E. Caten and J. H. Croft for criticisms and helpful comments during the preparation of the manuscript. B.G. and I.D.M. acknowledge receipt of S.R.C. studentships.

\section{REFERENCES}

Armit, S., McCullough, W. \& Roberts, C. F. (1976). Analysis of acetate non-utilizing (acu) mutants in Aspergillus nidulans. Journal of General Microbiology 92, 263-282.

Barratt, R. W., Johnson, G. B. \& OGata, W. N. (1965). Wild type and mutant stocks of Aspergillus nidulans. Genetics 52, 233-246.

Clutterbuck, A. J. (1974). Aspergillus nidulans. In Handbook of Genetics, vol. I, pp. 447-510. Edited by R. C. King. New York: Plenum Press.

Croft, J. H. \& Jinks, J. L. (I973). Genetical experiments with fungi. In Practical Genetics, pp. I73-224. Oxford: Blackwell.

Edwards, G. F. St. L., Normansell, I. D. \& Holt, G. (1975). Benlate induced haploidisation in diploid strains of Aspergillus nidulans and Penicillium chryosgenum. Aspergillus Newsletter I2, 12.

Giddings, B. \& UPShall, A. (1975). Spontaneous mapping of Aspergillus terreus and Aspergillus nidulans. Aspergillus Newsletter 13, I4. 
Hastie, A. C. (1970). Benlate-induced instability of Aspergillus diploids. Nature, London 226, 77.

KÄFER, E., MARShALl, P. \& CohEN, G. (1976). Well marked strains of Aspergillus nidulans for tests of environmental mutagens - identification of induced mitotic recombination and mutation. Mutation Research 38, 14I-146.

Kappas, A., Georgopoulos, S. G. \& Hastie, A. C. (1974). On the genetic activity of benzimidazole and thiophanate fungicides on diploid Aspergillus nidulans. Mutation Research 26, 17-27.

Mackintosh, M. E. \& Pritchard, R. H. (I963). The production and replica plating of micro-colonies of Aspergillus nidulans. Genetical Research 4, 320-322.

PONTECORVO, G. \& KäFER, E. (1958). Genetic analysis based on mitotic recombination. Advances in Genetics 9, 7I-IO4.

Pontecorvo, G., Roper, J. A., Hemmons, D. W., MacDonald, K. D. \& Bufton, A. W. J. (1953). The genetics of Aspergillus nidulans. Advances in Genetics 5, 141-238.

Pritchard, R. H. (1954). Ascospores with diploid nuclei in Aspergillus nidulans. Caryologia 6, Suppl. I, I I 17. RAPER, K. B. \& FENNELl, D. I. (1965). The Genus Aspergillus. Baltimore: Williams \& Wilkins.

ROPER, J. A. \& KäFER, E. (I957). Acriflavine resistant mutants of Aspergillus nidulans. Journal of General Microbiology $16,660-667$. 